\title{
Ropivacaína isolada ou associada à metadona ou à morfina, pela via epidural, em cadelas submetidas à ovário-histerectomia
}

\author{
[Ropivacaine sole or associated to methadone or morphine, by epidural route, in bitches undergoing \\ ovariohysterectomy] \\ N.R. Andrade ${ }^{1}$, B.C.P. Santos $^{2}$, L.P. Caires ${ }^{1}$, M.C. Azevedo ${ }^{3}$, E.F. Martins Filho ${ }^{4}$, \\ J.Q. Sande $e^{3}$, J.M. Costa Neto ${ }^{4}$, V.F. Barbosa ${ }^{4 *}$ \\ ${ }^{1}$ Aluno de pós-graduação - Universidade Federal da Bahia - Salvador, BA \\ ${ }^{2}$ Médico Veterinário - Salvador, BA \\ ${ }^{3}$ Aluno de residência - Universidade Federal da Bahia - Salvador, BA \\ ${ }^{4}$ Universidade Federal da Bahia - Salvador, BA
}

\section{RESUMO}

Objetivou-se avaliar os efeitos fisiológicos, sedativos e analgésicos da administração peridural de ropivacaína isolada ou associada à morfina ou à metadona. Para tal, 24 cadelas submetidas à ováriohisterectomia receberam acepromazina, e a anestesia foi induzida e mantida com propofol e isoflurano $\left(\mathrm{FiO}_{2}=1,0\right)$, respectivamente. De acordo com o protocolo peridural, formaram-se três grupos de igual número: GR (ropivacaína - 2,0mg/kg); GRMETA (ropivacaína $-2,0 \mathrm{mg} / \mathrm{kg}$ e metadona $-0,3 \mathrm{mg} / \mathrm{kg}$ ) e GRMORF (ropivacaína $-2,0 \mathrm{mg} / \mathrm{kg}$ e morfina $-0,1 \mathrm{mg} / \mathrm{kg}$ ). Registraram-se os parâmetros fisiológicos intraoperatórios e os graus de sedação e analgesia pós-operatórios. No GR constataram-se maiores médias de pressões arteriais 30 minutos após a anestesia epidural em relação ao GRMETA (sistólica e média) e ao final do procedimento cirúrgico comparativamente ao GRMORF (sistólica, diastólica e média). Não foram observadas diferenças significativas entre os grupos relativamente à analgesia e ao grau de sedação pós-operatórios. A administração epidural de ropivacaína é segura e eficaz e proporciona boa analgesia, independentemente da sua associação com morfina ou metadona.

Palavras-chave: cães, analgesia, anestesia, locorregional, opioides

\begin{abstract}
The aim of this study was to evaluate the physiological, sedative and analgesic effects of epidural administration of ropivacaine sole or associated to morphine or methadone. Twenty-four bitches were submitted to ovariohysterectomy and received acepromazine and after, propofol and isoflurane $\left(\mathrm{FiO}_{2}=\right.$ 1.0) for anesthesia induction and maintenance, respectively. Based on established epidural protocol (L7S1), three groups were formed: GR (ropivacaine - 2.0mg/kg); GRMETA (ropivacaine - 2.0mg/kg and methadone - $0.3 \mathrm{mg} / \mathrm{kg}$ ) and GRMORF (ropivacaine - $2.0 \mathrm{mg} / \mathrm{kg}$ and morphine - $0.1 \mathrm{mg} / \mathrm{kg}$ ). Intraoperative physiological parameters and degrees of postoperative sedation and analgesia were recorded. In the GR, the means of arterial pressures, 30 minutes after epidural anesthesia, were higher compared with GRMETA (systolic and mean) and, at the end of the clinical procedure, compared to GRMORF (systolic, diastolic and mean). Differences between groups were not observed for postoperative analgesia and degree of sedation. Epidural administration of ropivacaine is safe and effective and provides good analgesia regardless of its association with morphine or methadone.
\end{abstract}

Keywords: dogs, analgesia, anesthesia, locoregional, opioids

Recebido em 6 de novembro de 2017

Aceito em 17 de maio de 2018

*Autor para correspondência (corresponding author)

E-mail: vivian.fernanda@ufba.br 


\section{INTRODUÇÃO}

A crescente utilização de técnicas de anestesia balanceada visa primordialmente à obtenção de protocolos seguros, destacando-se a associação de fármacos opioides com anestésicos locais, seja no período pré ou transoperatório, objetivando-se ofertar analgesia e potencializar os anestésicos gerais, com redução de seus efeitos colaterais (Adami et al., 2012; Albuquerque et al., 2013; Diniz et al., 2013).

Técnicas anestésicas locorregionais são consideradas rotineiras na prática veterinária, destacando-se as administrações epidurais, cujas finalidades incluem bloqueio anestésico sensorial e motor; relaxamento muscular transoperatório; analgesia pós-cirúrgica e aprimoramento da qualidade da recuperação (Carvalho e Luna, 2007).

Por essa via, a ropivacaína destaca-se como alternativa à bupivacaína ao proporcionar menor bloqueio motor e toxicidades cardiovascular e nervosa, particularmente importante quando o risco de intoxicação sistêmica, por aplicação intravascular não intencional ou sobredosagem, é alto, a exemplo dos bloqueios nervosos epidurais ou periféricos (Leone et al., 2008; Beilin e Halpern, 2010).

Alguns estudos contemplando associações epidurais entre ropivacaína e opioides atestam estabilidade respiratória e do equilíbrio ácidobase, porém referem-se a efeitos adversos transitórios, como bradicardia, hipotermia e síndrome de Schiff-Sherrington (Silva et al., 2008; Albuquerque et al., 2013). A avaliação do uso epidural de ropivacaína associada ao sulfentanil, com ou sem epinefrina em cães, denotou aumento do bloqueio motor proporcional à dose do anestésico local e da analgesia pós-operatória similares nos grupos tratados com o opioide, descartando-se efeitos adversos relevantes (Adami et al., 2012).

Dessa forma, objetivou-se comparar a ação da ropivacaína, isolada ou associada à morfina ou à metadona, sobre parâmetros fisiológicos intraoperatórios, qualidade analgésica e grau de sedação pós-operatórios, bem como indicar o melhor protocolo, em cadelas submetidas à ovário-histerectomia $(\mathrm{OH})$.

\section{MATERIAL E MÉTODOS}

Este estudo foi aprovado pelo Comitê de Ética no Uso de Animais da Escola de Medicina Veterinária da Universidade Federal da Bahia (UFBA), sob protocolo de $n^{\circ} 47 / 2016$.

Foram selecionadas 24 cadelas provenientes da rotina cirúrgica do Hospital Veterinário da UFBA, Salvador, BA, com idade entre seis meses e três anos, e peso médio de $10,2 \pm 4,9 \mathrm{~kg}$, consideradas hígidas após exames físico e laboratoriais. Previamente ao procedimento anestésico, instituiu-se jejum hídrico e alimentar de duas e 12 horas, respectivamente, e, de acordo com o protocolo peridural avaliado, as fêmeas foram distribuídas aleatoriamente em três grupos experimentais, de oito animais cada, denominados: grupo ropivacaína (GR), grupo ropivacaína-metadona (GRMETA) e grupo ropivacaína-morfina (GRMORF).

Procedeu-se à tricotomia das regiões abdominal, lombossacral e sobre a veia cefálica, sendo essa última canulada para administração de solução de ringer lactato (Solução de Ringer Lactato, Halexistar, Brasil) na taxa de $10 \mathrm{~mL} / \mathrm{kg} / \mathrm{h}$. A acepromazina (Apromazin $1 \%{ }^{\circledR}$, Syntec do Brasil Ltda., Brasil) foi administrada como medicação pré-anestésica (MPA) na dose de $0,05 \mathrm{mg} / \mathrm{kg}$ IV. Decorridos 20 minutos, efetuou-se a indução anestésica com propofol (Diprivan $^{\circledR}$, AstraZeneca, Brasil), em dose suficiente para intubação orotraqueal $(5.2 \pm 2.4 \mathrm{mg} / \mathrm{kg}$ IV). Em seguida, iniciou-se a oferta de isoflurano (Isoforine $^{\circledR}$, Cristália, Brasil) com fração inspirada de oxigênio a $100 \% \quad\left(\mathrm{FiO}_{2}=1,0\right)$, mantendo-se as cadelas em ventilação espontânea.

Ato contínuo, os animais foram posicionados em decúbito esternal, com os membros pélvicos estendidos cranialmente e, após antissepsia com solução alcoólica de clorexidina $0,2 \%$, uma agulha de Tuohy (Procare ${ }^{\circledR}$, Lamedid, Brasil), apropriada ao porte da cadela, foi introduzida no espaço epidural lombossacro (L7-S1). O adequado posicionamento peridural da agulha foi confirmado pelo teste da aspiração de uma gota da solução anestésica, previamente posicionada no canhão da agulha, ou pela ausência de resistência à aplicação do(s) medicamento(s). Por essa via, nos animais do grupo GR, administrouse ropivacaína (Ropi ${ }^{\circledR} 0,75 \%$, Cristália, Brasil) 
na dose de $2 \mathrm{mg} / \mathrm{kg}$. As cadelas dos grupos GRMETA e GRMORF receberam o mesmo tratamento, acrescido de metadona (Mytedom ${ }^{\circledR}$, Cristália, Brasil), na dose de $0,3 \mathrm{mg} / \mathrm{kg}$, ou morfina (Dimorf ${ }^{\circledR}$, Cristália, Brasil), na dose de $0,1 \mathrm{mg} / \mathrm{kg}$. Nos três grupos, quando necessário, o volume infundido foi completado com solução de $\mathrm{NaCl}$ a $0,9 \%$, perfazendo-se $0,3 \mathrm{~mL} / \mathrm{kg}$, administrado na velocidade de $0,2 \mathrm{~mL} / \mathrm{s}$, para todos os animais.

Decorridos 30 minutos, as cadelas foram posicionadas em decúbito dorsal sobre colchão térmico ativo, mantendo-se a oferta de isoflurano em concentração suficiente para manutenção de plano cirúrgico de anestesia (estágio 3, plano 2), com ajustes estipulados entre 1,0 e 1,5CAM, durante todo o protocolo experimental, de acordo com mudanças na pressão arterial e/ou na frequência cardíaca (baseando-se no aumento ou na diminuição de 20\%), bem como nos sinais convencionais de anestesia profunda (perda do reflexo palpebral avaliado pelo toque suave dos cílios com o dedo e pelo posicionamento do globo ocular) (Mastrocinque et al., 2012).

Sequencialmente, procedeu-se à ováriohisterectomia $(\mathrm{OH})$ pela linha alba, estabelecendo-se para tal um tempo cirúrgico de 30 minutos. Ao longo do ensaio experimental, todos os avaliadores de variáveis foram cegos para os protocolos testados.

Foram aferidos, por meio de monitor multiparamétrico (Monitor Vita 400 ${ }^{\circledR}$, AlfaMed, Brasil), as variáveis: frequência cardíaca (FC); frequência respiratória $(f)$ e temperatura corporal $\left(\mathrm{T}^{\circ} \mathrm{C}\right)$, após posicionamento do sensor na ampola retal das cadelas, além da saturação de oxihemoglobina $\left(\mathrm{SpO}_{2}\right)$, mensurada por meio de oxímetro de pulso com o emissor/sensor adaptado à língua do animal. Adicionalmente, aferiu-se a pressão arterial sistólica (PAS) e a diastólica (PAD), por leitura em monitor de pressão oscilométrico veterinário (DLL $110^{\circledR}$, Deltamap, Brasil), cujo manguito de tamanho adequado fora posicionado no membro torácico esquerdo. Posteriormente, obteve-se a pressão arterial média (PAM) por meio de cálculo matemático, obedecendo-se à equação PAM = $\mathrm{PAD}+[(\mathrm{PAS}-\mathrm{PAD}) / 3]$, em que: PAM = pressão arterial média, $\mathrm{PAD}=$ pressão arterial diastólica e PAS = pressão arterial sistólica.
Tais parâmetros foram registrados nos seguintes momentos: 15 minutos após a MPA (M1), exceto para $\mathrm{SpO}_{2} ; 15$ minutos após a aplicação peridural (M2); 30 minutos após a aplicação peridural (M3); após o pinçamento do pedículo ovariano direito (M4); após pinçamento do pedículo ovariano esquerdo (M5); após pinçamento e ligadura do coto uterino (M6); após o início da sutura da cavidade abdominal (M7) e ao final da sutura de pele (M8).

O grau de sedação pós-operatório foi avaliado utilizando-se os seguintes escores: 0: animal alerta, respondendo prontamente aos estímulos externos; 1: animal levemente sedado - estado de relaxamento, calma e sonolência; 2: animal moderadamente sedado - estado de relaxamento e sonolência mais profundos, respondendo com dificuldade aos estímulos externos; 3: animal profundamente sedado (hipnose) - alheio ao ambiente, mas podendo responder lentamente aos estímulos externos (Maia, 2006).

A avaliação álgica pós-operatória foi realizada segundo escala de dor numérica, utilizando-se de variáveis objetivas (paramétricas) e subjetivas (não paramétricas) conforme Canôa et al. (2013), sendo atribuídos escores de 0 até 3 em cada variável, totalizando, ao final, entre 0 e 14 pontos (Fig. 1). Nessa somatória, valores compreendidos entre 0 e 5 foram interpretados como boa analgesia, entre 6 e 10, discreta analgesia e, acima de 10, nenhuma ou mínima analgesia. Para animais com somatório do escore igual ou superior a seis pontos, preconizou-se analgesia complementar com $2,0 \mathrm{mg} / \mathrm{kg}$ (IV) de tramadol (Tramadol $^{\circledR}$, Cristália, Brasil), o qual foi igualmente ministrado decorridas seis horas após o término da última avaliação álgica, nas cadelas que não necessitaram de analgesia complementar previamente.

Para determinação dos graus de sedação e analgesia, foram considerados os seguintes momentos pós-operatórios:

MP0 - imediatamente após a extubação;

MP1 - 60 minutos após o término da cirurgia; MP2 - 120 minutos após o término da cirurgia; MP4 - 240 minutos após o término cirúrgico e MP6 - 360 minutos após o término cirúrgico. 
Ropivacaína isolada ou associada...

\begin{tabular}{|c|c|c|}
\hline Parâmetro & Critério & Pontuação \\
\hline \multirow{4}{*}{$\mathrm{FC}, f$, PAS } & $</=10 \%$ maior que o valor pré-operatório & 0 \\
\hline & 11 a $30 \%$ maior que o valor pré-operatório & 1 \\
\hline & 31 a $50 \%$ maior que o valor pré-operatório & 2 \\
\hline & $50 \%$ maior que o valor pré-operatório & 3 \\
\hline \multirow{2}{*}{ Salivação } & Normal & 0 \\
\hline & Acima do normal & 1 \\
\hline \multirow{2}{*}{ Pupilas } & Normal & 0 \\
\hline & Dilatadas & 1 \\
\hline \multirow{3}{*}{ Vocalização } & Sem vocalização & 0 \\
\hline & Vocalização presente e controlada sem medicação & 1 \\
\hline & Vocalização presente não controlada & 2 \\
\hline \multirow{4}{*}{ Agitação } & Adormecido ou calmo & 0 \\
\hline & Leve agitação & 1 \\
\hline & Moderada agitação & 2 \\
\hline & Severa agitação & 3 \\
\hline \multirow[b]{2}{*}{ Postura } & Decúbito esternal ou movendo-se com tranquilidade & 0 \\
\hline & $\begin{array}{l}\text { Defendendo e protegendo a área afetada, incluindo posição } \\
\text { fetal }\end{array}$ & 1 \\
\hline \multirow{4}{*}{$\begin{array}{l}\text { Resposta à } \\
\text { manipulação da } \\
\text { ferida cirúrgica }\end{array}$} & Sem resposta & 0 \\
\hline & Resposta mínima, tenta esquivar-se & 1 \\
\hline & Vira cabeça em direção à ferida cirúrgica, leve vocalização & 2 \\
\hline & Vira a cabeça com intenção de morder, severa vocalização & 3 \\
\hline
\end{tabular}

Adaptado de Canôa et al. (2013).

Figura 1. Critérios utilizados para avaliação do grau de analgesia.

Complementarmente, foram registrados: o período hábil, compreendido entre o estabelecimento do período de latência e o retorno do paciente à posição quadrupedal com deambulação normal; os tempos de duração do relaxamento do esfíncter anal, do relaxamento da cauda e da perda do reflexo interdigital; e a ocorrência de micção ou defecação espontânea imediatamente após a aplicação peridural. Todas as cadelas receberam $0,2 \mathrm{mg} / \mathrm{kg}$ (SC) de meloxicam (Maxicam $^{\circledR}$, Ourofino, Brasil) e $5,0 \mathrm{mg} / \mathrm{kg}$ (SC) de enrofloxacina $\left(\right.$ Chemtril $^{\circledR}$, Chemitec, Brasil) após o término da avaliação experimental.

Os dados foram submetidos ao teste de Kolmogorov-Smimov para verificação da normalidade de distribuição e homogeneidade das amostras. Os valores das variáveis fisiológicas e dos graus de sedação e de analgesia foram analisados utilizando-se o teste análise de variância, e as médias foram comparadas por meio do teste de Tukey. As variáveis de tempo (período hábil; durações do relaxamento do esfíncter anal, do relaxamento da cauda e da perda do reflexo interdigital) foram submetidas ao teste de Friedman, seguido do teste de Dunn, para comparações múltiplas. Os registros de micção e defecção foram analisados utilizandose o teste exato de Fisher. As análises foram realizadas mediante o uso do software Prism 5.0 (GraphPad, San Diego, CA, EUA), considerando-se um nível de significância de $5 \%$.

\section{RESULTADOS E DISCUSSÃO}

Para melhor visualização dos resultados, os valores médios e os desvios-padrão das variáveis fisiológicas foram apresentados em forma tabular (Tab. 1).

Iniciando-se pelas variáveis cardiovasculares, a FC não foi influenciada pelos diferentes protocolos testados $(\mathrm{P}=0,081)$. Contudo, foram constatadas diminuições em relação ao momento inicial (M1), no grupo GRMETA, 30 minutos após a aplicação peridural e, no grupo GRMORF, após ligadura do coto uterino. Tais variações coincidem com os períodos de latência 
dos opioides utilizados e refletem possivelmente suas ações simpatolíticas (Jones, 2001; Leibetseder et al., 2006). De acordo com Murrell (2011), doses de metadona até $1,0 \mathrm{mg} / \mathrm{kg}$, em cães e gatos, não estão associadas a efeitos cardiovasculares adversos, todavia reduções das frequências cardíaca e respiratória podem ocorrer em animais anestesiados. Vale salientar, entretanto, que as médias permanecem dentro do intervalo de referência para a espécie canina, não denotando significado clínico (Redondo et al., 2007). Complementarmente, a avaliação da administração epidural de ropivacaína associada a duas diferentes doses de morfina, em cadelas submetidas à $\mathrm{OH}$, não retratou variação entre os tratamentos sobre a frequência e o ritmo cardíacos que, igualmente, mantiveram-se em níveis fisiológicos (Albuquerque et al., 2013).

A análise das pressões arteriais revelou valores reduzidos para o GRMETA (PAS e PAM) e o GRMORF (PAS, PAM e PAD) em relação ao GR, 30 minutos após a aplicação peridural (M3), no grupo que recebeu metadona e, ao final da sutura de pele (M8), no grupo tratado com morfina. Nota-se ainda, nos grupos tratados com opioides, redução mais acentuada das médias ao longo do tempo. A associação epidural de ropivacaína com metadona, em cães, resultou em menor pressão arterial sistêmica em relação ao uso de salina ou de metadona isoladamente (Bosmans et al., 2011). É sabido que os anestésicos locais, quando administrados no espaço peridural, podem induzir hipotensão, bloqueando os eferentes simpáticos préganglionares e, assim, reduzindo a atividade vasoconstrictora nas áreas afetadas. A magnitude desse efeito depende do equilíbrio entre a atividade simpática e a parassimpática, da extensão do bloqueio simpático, do efeito dos anestésicos locais sistematicamente absorvidos, do grau de enchimento cardíaco e da adequação da função cardíaca (Veering e Cousins, 2000).

Com relação ao maior efeito hipotensor nos grupos que receberam opioide, alguns autores referem-se à ação vasodilatadora tanto com o uso da morfina quanto da metadona, devido principalmente à potencial liberação de histamina promovida por esses fármacos (Bowdle et al., 2004). A comparação do uso epidural da morfina isolada ou associada à bupivacaína, em cães submetidos a cirurgias ortopédicas, revelou menores valores de pressão arterial mediante a combinação de agentes, corroborando o estudo em pauta (Kona-Boun et al., 2006). Além disso, a avaliação da combinação de lidocaína associada ao fentanil ou à metadona, em cadelas submetidas à $\mathrm{OH}$, mostrou redução significativa da PAS com ambos os protocolos, porém vale salientar que as médias permaneceram maiores que as deste ensaio, visto que os animais ficaram despertos durante o procedimento cirúrgico (Diniz et al., 2013). Tal fato ratifica a influência dos anestésicos gerais utilizados sobre a resistência vascular periférica (Adams et al., 1994) e a necessidade de considerar o uso de medidas terapêuticas para o controle da hipotensão diante das associações epidurais de opioides e anestésicos locais.

Relativamente aos parâmetros respiratórios, a $f$ não foi influenciada pelos protocolos testados, no entanto apresentou redução em relação ao momento inicial de avaliação até valores próximos à normalidade em todos os grupos, fato que pode ser imputado às ações simpatolíticas do propofol e do isoflurano (Adams et al., 1994). Para a $\mathrm{SpO}_{2}$, com exceção do momento M6, no qual houve menores médias no GRMORF em relação ao GRMETA $(\mathrm{P}=0,0439)$, não foram detectadas variações ao longo dos momentos. Classicamente, opioides com ação agonista mu estão vinculados à ocorrência de depressão respiratória proporcional à dose administrada. Dentro desse conceito, as pequenas doses utilizadas pela via peridural tendem a minimizar tais repercussões (Valadão et al., 2002). Todavia, a manutenção da $\mathrm{SpO}_{2}$ em níveis fisiológicos (Redondo et al., 2007), possivelmente em virtude da oferta de oxigênio a $100 \%$ e da manutenção dos mecanismos de perfusão, permite descartar a ocorrência de episódios hipoxêmicos independentemente do protocolo testado.

Embora isenta de variação entre tratamentos (P>0,6908), a $\mathrm{T}^{\circ} \mathrm{C}$ se reduziu de forma gradativa, comparando-se aos valores basais, 15 minutos após realização da aplicação peridural (M2) para os grupos GRMORF e GRMETA e 30 minutos pós-peridural (M3) para o grupo GR. Tais registros provavelmente relacionam-se à associação de fármacos utilizados, destacando-se a acepromazina, que promove vasodilatação, suprime $\mathrm{o}$ tremor $\mathrm{e}$ permite diminuição rápida da temperatura, inibindo as defesas termorreguladoras (Liu et al., 
2015). Os achados estão de acordo com Albuquerque et al. (2013), que observaram redução térmica gradual ao utilizarem ropivacaína isolada ou associada à morfina, ao butorfanol ou ao tramadol, pela via peridural, em cadelas submetidas à $\mathrm{OH}$.

Tabela 1. Valores médios $(\overline{\mathbf{x}})$ e desvios-padrão $( \pm \sigma)$ da FC (bpm), PAS (mmHg), PAD (mmHg), PAM $(\mathrm{mmHg}), f(\mathrm{mpm}), \mathrm{SpO}_{2}$ e $\mathrm{T}^{\circ} \mathrm{C}$ de cadelas submetidas à ovário-histerectomia, tratadas com ropivacaína isolada (GR) ou associada à metadona (GRMETA) ou à morfina (GRMORF), pela via epidural, anestesiadas com isoflurano

\begin{tabular}{|c|c|c|c|c|c|c|c|c|c|}
\hline Variável & Grupo & \multicolumn{8}{|c|}{ Momentos } \\
\hline \multirow{3}{*}{$\mathrm{FC}(\mathrm{bpm})$} & GR & $108 \pm 14$ & $93 \pm 11$ & $94 \pm 7,0$ & $103 \pm 11$ & $98 \pm 9,2$ & $95 \pm 11$ & $96 \pm 17$ & $95 \pm 17$ \\
\hline & GRMETA & $108 \pm 19^{\mathrm{a}}$ & $85 \pm 14^{\mathrm{ab}}$ & $85 \pm 12^{b}$ & $96 \pm 11^{\mathrm{ab}}$ & $100 \pm 11^{b}$ & $88 \pm 8,1^{\mathrm{ab}}$ & $88 \pm 5,0^{\mathrm{ab}}$ & $92 \pm 14^{\mathrm{ab}}$ \\
\hline & GR & $116 \pm 10$ & $93 \pm 20$ & $93 \pm 6,1^{\mathrm{A}}$ & $97 \pm 21$ & $107 \pm 24$ & $96 \pm 21$ & $87 \pm 21$ & $97 \pm 14^{\mathrm{A}}$ \\
\hline \multirow{2}{*}{$\begin{array}{c}\text { PAS } \\
(\mathrm{mmHg})\end{array}$} & GRMORF & $122 \pm 15^{a}$ & $82 \pm 5,7^{b}$ & $82 \pm 13^{\mathrm{ABb}}$ & $100 \pm 15^{a}$ & $88 \pm 5,7^{\mathrm{b}}$ & $82 \pm 15^{\mathrm{b}}$ & $82 \pm 18^{\mathrm{b}}$ & $81 \pm 11^{\mathrm{Bb}}$ \\
\hline & GR & $85 \pm 13^{a}$ & $56 \pm 16^{\mathrm{b}}$ & $59 \pm 12^{\mathrm{b}}$ & $62 \pm 20^{\mathrm{ab}}$ & $70 \pm 22^{\mathrm{ab}}$ & $59 \pm 17^{\mathrm{ab}}$ & $55 \pm 17^{\mathrm{b}}$ & $64 \pm 14^{\mathrm{Ab}}$ \\
\hline \multirow{3}{*}{$\begin{array}{c}\mathrm{PAD} \\
(\mathrm{mmHg})\end{array}$} & GRMORF & $80 \pm 17^{a}$ & $42 \pm 9,2^{b}$ & $47 \pm 13^{b}$ & $63 \pm 15^{\mathrm{ab}}$ & $59 \pm 18^{\text {ab }}$ & $43 \pm 14^{b}$ & $45 \pm 15^{\mathrm{b}}$ & $39 \pm 3,9^{\mathrm{Bb}}$ \\
\hline & GRMETA & $88 \pm 17^{\mathrm{a}}$ & $48 \pm 13^{\mathrm{b}}$ & $42 \pm 20^{\mathrm{b}}$ & $54 \pm 14^{\mathrm{b}}$ & $60 \pm 20^{\mathrm{ab}}$ & $51 \pm 18^{\mathrm{ab}}$ & $39 \pm 4,0^{b}$ & $50 \pm 7,9^{\mathrm{ABb}}$ \\
\hline & GR & $96 \pm 16^{a}$ & $68 \pm 16^{\mathrm{b}}$ & $73 \pm 8,9^{\mathrm{Ab}}$ & $75 \pm 19^{\mathrm{ab}}$ & $83 \pm 24^{\mathrm{ab}}$ & $73 \pm 19^{\mathrm{ab}}$ & $67 \pm 19^{\mathrm{ab}}$ & $76 \pm 13^{\text {Aab }}$ \\
\hline \multirow{2}{*}{$\begin{array}{c}\text { PAM } \\
(\mathrm{mmHg})\end{array}$} & GRMORF & $92 \pm 15^{a}$ & $55 \pm 11^{\mathrm{ab}}$ & $59 \pm 10^{\mathrm{ABab}}$ & $77 \pm 17^{\mathrm{ab}}$ & $69 \pm 18^{\mathrm{ab}}$ & $56 \pm 1^{\mathrm{b}}$ & $58 \pm 11^{\mathrm{b}}$ & $56 \pm 14^{\mathrm{Bab}}$ \\
\hline & GRMETA & $99 \pm 20^{a}$ & $59 \pm 14^{\mathrm{b}}$ & $47 \pm 13^{\mathrm{Bb}}$ & $66 \pm 14^{\mathrm{ab}}$ & $70 \pm 20^{\mathrm{ab}}$ & $61 \pm 18^{b}$ & $51 \pm 6,0^{\mathrm{b}}$ & $64 \pm 16^{\mathrm{ABb}}$ \\
\hline \multirow{3}{*}{$\mathrm{SpO}_{2}$} & GRMORF & - & $97 \pm 1,4$ & $97 \pm 2,1$ & $97 \pm 2,2$ & $97 \pm 2,5$ & $97 \pm 2,8^{\mathrm{A}}$ & $97 \pm 2,3$ & $97 \pm 2,1$ \\
\hline & GRMETA & - & $98 \pm 1,9$ & $97 \pm 2,7$ & $99 \pm 1,1$ & $99 \pm 0,74$ & $98 \pm 1,2^{\mathrm{B}}$ & $99 \pm 1,8$ & $99 \pm 1,8$ \\
\hline & GR & $38 \pm 0,26^{\mathrm{a}}$ & $37 \pm 0,71^{\mathrm{ab}}$ & $37 \pm 0,91^{\mathrm{b}}$ & $36 \pm 1,1^{\mathrm{bc}}$ & $36 \pm 1,2^{\mathrm{bc}}$ & $36 \pm 1,3^{\mathrm{bc}}$ & $35 \pm 1,2^{\mathrm{bc}}$ & $35 \pm 1,3^{c}$ \\
\hline \multirow{2}{*}{$\mathrm{T}^{\circ} \mathrm{C}$} & GRMORF & $38 \pm 0,48^{\mathrm{a}}$ & $37 \pm 0,91^{\mathrm{b}}$ & $37 \pm 0,99^{\mathrm{bc}}$ & $36 \pm 1,0^{\mathrm{bc}}$ & $36 \pm 1,0^{\mathrm{bc}}$ & $36 \pm 1,0^{\mathrm{bc}}$ & $36 \pm 0,91^{b c}$ & $35 \pm 0,81^{\mathrm{c}}$ \\
\hline & GRMETA & $38 \pm 0,46^{\mathrm{a}}$ & $37 \pm 0,71^{\mathrm{b}}$ & $37 \pm 0,73^{\mathrm{bc}}$ & $36 \pm 0,79^{b c}$ & $36 \pm 0,85^{\mathrm{bc}}$ & $36 \pm 0,84^{\mathrm{c}}$ & $35 \pm 0,84^{\mathrm{c}}$ & $35 \pm 0,88^{c}$ \\
\hline
\end{tabular}

Médias seguidas por letras maiúsculas diferentes, nas colunas, demonstram diferença entre grupos $(\mathrm{P}<0,05)$. Médias seguidas por letras minúsculas diferentes, nas linhas, demonstram diferença entre momentos $(\mathrm{P}<0,05)$.

No que diz respeito à duração de efeito peridural (Tab. 2), o grupo tratado com morfina apresentou menores tempos de recuperação dos tônus de esfíncter anal e de cauda em relação ao GR e ao GRMETA. A literatura se refere a um possível efeito "diluidor" de alguns opioides sobre o anestésico local, quando associados (Silva et al., 2008), considerando-se essa a melhor hipótese para tais achados. Há que se considerar, ainda, que o volume farmacológico peridural foi calculado com base no peso das cadelas e não no comprimento da coluna vertebral, o que pode interferir no tempo hábil e na extensão do bloqueio (Leite et al., 2017). Dentro desse conceito, pode-se considerar como hipótese uma possível variação individual do perfil anatômico mais longilíneo dos animais do GRMORF, que poderia implicar a distribuição do anestésico local no canal medular e, consequentemente, na duração do bloqueio de alguns segmentos. Contudo, a inclusão de opioides não foi capaz de interferir nos tempos de recuperação da deambulação e de reestabelecimento do reflexo interdigital.

Não houve variação para ocorrência de micção $(\mathrm{P}=0,0727)$ e defecação $(\mathrm{P}=0,2397)$ entre os grupos, constatando-se, contudo, elevada frequência desses registros para o GR $(62,5 \%$ e $37,5 \%)$, o GRMETA $(50 \%$ e $62,5 \%)$ e o GRMORF (100\% e 25\%). A hipótese mais plausível seria os estados da bexiga e do cólon repletos dos animais, uma vez que estes permaneceram sem caminhar por longos períodos previamente ao protocolo experimental, conforme também fora observado por Maia (2006).

Aos 60 minutos após o término cirúrgico, todos os animais tratados com metadona apresentaramse alertas (grau de sedação $=0$ ), fato observado aos 120 minutos pós-cirúrgicos em $87,5 \%$ das cadelas do GR e do GRMORF, e em 100\% dos animais aos 240 minutos pós-cirúrgicos, descartando-se variação estatística significativa. 
Assim, sugere-se que a disposição precoce dos animais tratados com metadona reflete maior conforto imediato proporcionado por esse opioide, uma vez que, devido ao seu caráter lipofílico e à menor latência, este se apresentava mais biodisponível sistemicamente que a morfina no período inicial de avaliação (Valverde, 2008).

Tabela 2. Valores médios $(\square)$ e desvios-padrão $( \pm \sigma)$ dos tempos para restabelecimento, em minutos, do tônus de esfíncter anal, do tônus caudal, de reflexo interdigital e da deambulação normal de cadelas submetidas à ovário-histerectomia, tratadas com ropivacaína isolada (GR) ou associada à metadona (GRMETA) ou à morfina (GRMORF), pela via epidural, anestesiadas com isoflurano

\begin{tabular}{cccc}
\hline & \multicolumn{3}{c}{ Tempo para restabelecimento } \\
\cline { 2 - 4 } Variável & GR & GRMORF & GRMETA \\
\hline Tônus de esfincter anal & $372,10 \pm 33,19^{\mathrm{A}}$ & $179,00 \pm 33,42^{\mathrm{B}}$ & $287,50 \pm 68,12^{\mathrm{C}}$ \\
Tônus caudal & $345,50 \pm 30,98^{\mathrm{A}}$ & $187,00 \pm 59,47^{\mathrm{B}}$ & $246,30 \pm 58,61^{\mathrm{C}}$ \\
Reflexo interdigital & $347,60 \pm 61,47$ & $212,80 \pm 123,10$ & $290,40 \pm 117,00$ \\
Deambulação normal & $437,60 \pm 75,81$ & $405,30 \pm 213,00$ & $405,30 \pm 142,70$
\end{tabular}

Médias seguidas por letras maiúsculas diferentes, nas linhas, demonstram diferença estatística significativa entre grupos $(\mathrm{P}<0,05)$.

No que tange à avaliação analgésica pósoperatória, não foram constatadas diferenças. Verificou-se boa analgesia nos diferentes grupos, obtendo-se em todos os momentos somatório do escore (pontuação final) igual ou menor que 4 pontos (Tab. 3).

Tabela 3. Pontuação final relativa ao grau de analgesia de acordo com o momento pós-cirúrgico (MP0; MP1; MP2; MP4 e MP6) de cadelas submetidas à ovário-histerectomia, tratadas com ropivacaína isolada (GR) ou associada à metadona (GRMETA) ou à morfina (GRMORF), pela via epidural, anestesiadas com isoflurano

\begin{tabular}{|c|c|c|c|c|}
\hline \multirow{2}{*}{ Momentos } & \multirow{2}{*}{$\begin{array}{l}\text { Pontuação } \\
\text { Final }\end{array}$} & \multicolumn{3}{|c|}{ Número de animais } \\
\hline & & GR & GRMORF & GRMETA \\
\hline \multirow{5}{*}{ MP0 } & 0 & $5(62,5 \%)$ & $7(87,5 \%)$ & $6(75,0 \%)$ \\
\hline & 1 & $3(37,5 \%)$ & $1(12,5 \%)$ & $*$ \\
\hline & 2 & $*$ & $*$ & $1(12,5 \%)$ \\
\hline & 3 & $*$ & $*$ & $1(12,5 \%)$ \\
\hline & 4 & $*$ & $*$ & $*$ \\
\hline \multirow{5}{*}{ MP1 } & 0 & $7(87,5 \%)$ & $6(75,0 \%)$ & $6(75,0 \%)$ \\
\hline & 1 & $1(12,5 \%)$ & $1(12,5 \%)$ & $*$ \\
\hline & 2 & $*$ & $1(12,5 \%)$ & $*$ \\
\hline & 3 & $*$ & $*$ & $1(12,5 \%)$ \\
\hline & 4 & $*$ & $*$ & $1(12,5 \%)$ \\
\hline \multirow{5}{*}{ MP2 } & 0 & $7(87,5 \%)$ & $7(87,5 \%)$ & $6(75,0 \%)$ \\
\hline & 1 & $1(12,5 \%)$ & $1(12,5 \%)$ & $1(12,5 \%)$ \\
\hline & 2 & $*$ & $*$ & $1(12,5 \%)$ \\
\hline & 3 & $*$ & $*$ & $*$ \\
\hline & 4 & * & * & * \\
\hline \multirow{5}{*}{ MP4 } & 0 & $8(100,0 \%)$ & $8(100,0 \%)$ & $7(87,5 \%)$ \\
\hline & 1 & $*$ & $*$ & $*$ \\
\hline & 2 & $*$ & $*$ & $1(12,5 \%)$ \\
\hline & 3 & $*$ & $*$ & $*$ \\
\hline & 4 & $*$ & $*$ & $*$ \\
\hline \multirow{5}{*}{ MP6 } & 0 & $8(100,0 \%)$ & $8(100,0 \%)$ & $8(100,0 \%)$ \\
\hline & 1 & * & $*$ & $*$ \\
\hline & 2 & $*$ & $*$ & $*$ \\
\hline & 3 & $*$ & $*$ & $*$ \\
\hline & 4 & $*$ & * & $*$ \\
\hline
\end{tabular}


Vale ressaltar que, aos 60 minutos pós-cirúrgicos (MP0), dois animais do grupo GRMETA apresentaram escores 3 e 4 de analgesia, fato provavelmente vinculado a uma maior sensibilidade individual dessas cadelas. Tal hipótese reveste-se de significado ao se considerar que todos os animais ainda permaneciam sob o efeito do anestésico local, cuja literatura se refere à duração de 180 a 480 minutos (Leone et al., 2008). Considerando-se que a maioria das cadelas tenha demonstrado conforto analgésico pós-operatório e que se tenha detectado ausência completa de dor aos 360 minutos pós-cirúrgicos (MP4), em todos os grupos avaliados, pode-se afirmar que, ao longo desse período, a inclusão dos opioides testados não interferiu na eficácia analgésica da ropivacaína.

\section{CONCLUSÕES}

A associação de morfina ou metadona à ropivacaína, pela via epidural, não resultou em alterações fisiológicas transoperatórias relevantes, porém requer monitoração e, se necessário, controle da pressão arterial. A ropivacaína proporcionou boa qualidade analgésica e baixo grau de sedação póscirúrgicos, independentemente da inclusão de morfina ou metadona ao protocolo epidural.

\section{AGRADECIMENTOS}

O presente trabalho foi realizado com apoio da Coordenação de Aperfeiçoamento de Pessoal de Nível Superior - Brasil (CAPES) - Código de Financiamento 001

\section{REFERÊNCIAS}

ADAMI, C.; VERES-NYÉKI, K.; SPADAVECCHIA, C. et al. Evaluation of peri-operative epidural analgesia with ropivacaine, ropivacaine and sufentanil, and ropivacaine, sufentanil and epinephrine in isoflurane anesthetized dogs undergoing tibial plateau levelling osteotomy. Vet. J., v.194, p.229-234, 2012.

ADAMS, H.A.; SCHMITZ, C.S.; BALTESGOTZ, B. Propofol vs isoflurane-endocrine stress-response, hemodynamic reaction, and recovery after total intravenous and inhalation anesthesia. Anaesthesia, v.43, p.730-737, 1994.
ALBUQUERQUE, V.B.; SOUZA, T.F.B.; VIVAN, M.C.R. et al. Ropivacaína isolada ou associada à morfina, butorfanol ou tramadol pela via peridural em cadelas para realização de ovariosalpingohisterectomia. Vet. Zootec., v.20, p.111-123, 2013.

BEILIN, Y.; HALPERN, S. Ropivacaine versus bupivacaine for epidural labor analgesia. Anesth. Analg., v.111, p.482-487, 2010.

BOSMANS, T.; SCHAUVLIEGE, S.; GASTHUYS, F. et al. Cardiovascular effects of epidural administration of methadone, ropivacaine $0.75 \%$ and their combination in isoflurane anaesthetized dogs. Vet. Anaesth. Analg., v.38, p.146-157, 2011.

BOWDLE, T.A.; EVEN, A.; SHEN, D.D. et al. Methadone for the induction of anesthesia: plasma histamine concentration, arterial blood pressure, and heart rate. Anesth. Analg., v.98, p.1692-1697, 2004.

CANÔA, J.T.B.; ALBUQUERQUE V.B.; OLIVEIRA, G.C.V. et al. Analgesia peridural com ropivacaína associada ao midazolam em cadelas submetidas à ovariosalpingohisterectomia. Vet. Zootec., v.20, p.326-337, 2013.

CARVALHO, Y.K.; LUNA, S.P.L. Anestesia e analgesia por via epidural em cães - atualização farmacológica para uma técnica tradicional. Clín. Vet., v.12, p.68-76, 2007.

DINIZ, M.S.; KANASHIRO, G.P.; BERNARDI, C.A. et al. Extradural anestesia with lidocaine combined with fentanyl or methadone to ovariohisterectomy in dogs. Acta Cir. Bras., v.28, p.532-535, 2013.

JONES, R.S. Epidural analgesia in the dog and cat. J. Vet., v.161, p.123-131, 2001.

KONA-BOUN, J.J.Ç.; CUVELLIEZ, S.; TRONCY, E. Evaluation of epidural administration of morphine or morphine and bupivacaine for postoperative analgesia after premedication with an opioid analgesic and orthopedic surgery in dogs. J. Am. Vet. Med. Assoc., v.229, p.1103-1112, 2006.

LEIBETSEDER, E.N.; MOSING, M.; JONES, R.S. A comparison of extradural and intravenous methadone on intraoperative isoflurane and postoperative analgesia requirements in dogs. Vet. Anaesth. Analg., v.33, p.128-136, 2006. 
LEITE, G.M.D.P.; AUGUSTO, L.E.F; PEREIRA, V.G. et al. Comparative study of epidural anesthesia in dogs by weight or occipito-coccygeal distance. Rev. Ceres, v.64, p.12-17, 2017.

LEONE, S.; DI CIANNI, S.; CASATI, A. et al. Pharmacology, toxicology, and clinical use of new long acting local anesthetics, ropivacaine and levobupivacaine. Acta Biomed., v.79, p.92105,2008

LIU, S.; GENG, X.; FORREIDER, B. et al. Enhanced beneficial effects of mild hypothermia by phenothiazine drugs in stroke therapy. Neurol. Res., v.37, p.454-460, 2015.

MAIA, C.A.A. Avaliação cardiorrespiratória e analgésica da ropivacaína associada à morfina, petidina ou butorfanol administrados pela via peridural em cães. 2006. 114f. Dissertação (Mestrado em Anestesiologia Experimental) Faculdade de Medicina, Universidade Estadual Paulista, Botucatu, SP.

MASTROCINQUE, S.; ALMEIDA, T.F.; TATARUNAS, A.C. et al. Comparison of epidural and systemic tramadol for analgesia following ovariohysterectomy. J. Am. Anim. Hosp. Assoc., v.48, p.310-319, 2012.
MURRELL, J. Clinical use of methadone in cats and dogs. Comp. Anim., v.16, p.56-61, 2011.

REDONDO, J.I.; RUBIO, M.; SOLER, G. et al. Normal values and incidence of cardiorespiratory complications in dogs during general anaesthesia. A review of 1281 cases. J. Vet. Med. Assoc., v.54, p.470-477, 2007.

SILVA, B.M.; MATSUBARA, L.M.; ALBUQUERQUE, V.B. et al. Ropivacaína isolada e associada ao fentanil ou ao tramadol administrador pela via peridural em cães. Cienc. Rural, v.38, p.2197-2202, 2008.

VALADÃO, C.A.A.; DUQUE, J.C.; FARIAS, A. Administração epidural de opioides em cães. Cienc. Rural, v.32, p.347-355, 2002.

VALVERDE, A. Epidural analgesia and anesthesia in dogs and cats. Vet. Clin. N. Am. Small Anim. Pract., v.38, p.1205-1230, 2008.

VEERING, B.T.; COUSINS, M.J. Cardiovascular and pulmonary effects of epidural anaesthesia. Anaesth. Intensive Care, v.28, p.620-635, 2000. 